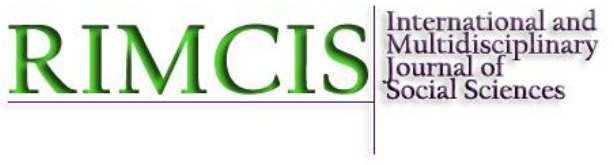

Hipatia Press

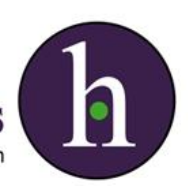

Instructions for authors, subscriptions and further details:

http://rimcis.hipatiapress.com

\title{
Antropología, Historia y las Instituciones Socioculturales como Nexo de Unión entre Ambas. Otro Apunte Más para su Discusión
}

Ignacio Alcalde Sánchez ${ }^{1}$

1) University of Cordoba, Spain

Date of publication: July $30^{\text {th }}, 2019$

Edition period: July 2019 - November 2019

To cite this article: Alcalde Sánchez, I. (2019). Antropología, Historia y las Instituciones Socioculturales como Nexo de Unión entre Ambas. Otro Apunte Más para su Discusión. International and Multidisciplinary Journal of Social Sciences, 8(2), 126-145. doi: 10.17583/rimcis.2019.4072

To link this article: http://doi.org/10.17583/rimcis.2019.4072

\section{PLEASE SCROLL DOWN FOR ARTICLE}

The terms and conditions of use are related to the Open Journal System and to Creative Commons Attribution License (CC-BY). 


\section{Anthropology, History and the Sociocultural Institutions as a Link between Them. Another Contribution on the Discussion}

Ignacio Alcalde Sánchez

University of Cordoba

\section{Abstract}

Is the Anthropology an auxiliary science of the History? Is the History an empty explanation of the facts? Can we define the Anthropology as the History of the nonhistory communities? Or is the History only the science of the winners? In this article we review the permanent controversial relation between History and Anthropology as sciences with a common way. We show the main theoretic concepts to argue the different points of view, in one or another direction and, at the same time, we realize a brief historic approach about the different stages of this dialogue. We show as well, the different common fields they have got and finally, we propose a new link to work together under the sociocultural institutions approach. They can be very useful as a tool to establish a new bridge to connect both sciences. We try to expose a theoretic revision to enrich the present analysis that we complete with some current related researches.

Keywords: Anthropology, History, Etnohistory, institutions, oral history 


\section{Antropología, Historia y las Instituciones Socioculturales como Nexo de Unión entre}

\section{Ambas. Otro Apunte Más para su Discusión}

Ignacio Alcalde Sánchez

University of Cordoba

\section{Resumen}

¿La Antropología como ciencia auxiliar de la Historia? ¿La Historia como versión vacía de los hechos? ¿La Antropología como la Historia de los pueblos sin historia? $\mathrm{O}$ ¿la Historia como la ciencia de los vencedores? En este artículo revisamos la siempre polémica relación existente entre Historia y Antropología como ciencias con una trayectoria común. Mostramos los conceptos teóricos con los que se argumenta tanto su trabajo conjunto y coordinación como su separación; al tiempo que realizamos un breve recorrido histórico por las diferentes etapas que ha atravesado este diálogo y las diferentes ramas de ambas que sí parecen encontrarse. Finalmente proponemos un nuevo nexo de unión bajo el prisma de las instituciones socioculturales, sirviendo éstas como herramientas con las que establecer un nuevo puente de entendimiento entre ambas. Un aporte más a la revisión teórica de ambas ciencias con las que enriquecer el debate actual que terminaremos con la muestra de algunos estudios recientes en esta línea.

Palabras clave: Antropología, Historia, etnohistoria, instituciones, historia oral

2019 Hipatia Press

ISSN: 2014-3680

DOI: $10.17583 /$ rimcis.2019.4072 


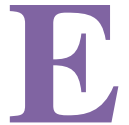

ste artículo nace con la idea de aportar otra pincelada más al debate constante que existe en la relación entre la Antropología y la Historia. Una revisión sobre el desarrollo teórico que plantea su combinación o exclusión y un análisis de las diferencias y semejanzas entre éstas, así como una posterior propuesta de la conveniencia de su coordinación a través de los estudios de las instituciones socioculturales, utilizadas a modo de herramientas de análisis. Reflejándolo finalmente, a modo de ejemplo, en algunos estudios en los que ambas ciencias se entrecruzan en su análisis de las instituciones contemporáneas.

Para ello, describiremos la utilidad de la Antropología para la Historia y a la inversa, en el análisis de las instituciones socioculturales y expondremos cómo ambas ciencias han realizado recorridos distintos para poder encontrarse en la actualidad y trabajar de manera coordinada.

De esta manera, mostramos aquí cómo ambas ciencias comparten nexos en común a través de un análisis sobre su fundamentación y un breve recorrido por sus últimas etapas como ciencias para, después, mostrar a modo de aplicación práctica, algunos ejemplos en los que los estudios etnográficos e históricos se combinan, enriqueciéndose mutuamente.

Realizaremos así, una aproximación entre la Antropología y la Historia con la que exponer la conveniencia del análisis histórico para el estudio etnográfico-antropológico y a la inversa, la utilidad de la Antropología para la Historia en las reconstrucciones historiográficas. Partimos, por tanto, lejos de querer delimitar o avivar el debate sobre la relación entre ambas ciencias y aún menos de intentar arrojar argumentos definitivos en este campo, de la intención de mostrar de manera práctica la utilidad de coordinar ambas ciencias en el marco de la investigación. En un momento como el actual, en el que la transversalidad y la multidisciplinariedad se muestran como la pauta a seguir, no habría cabida para ese enfoque unívoco y simplista por el que estas ciencias caminasen de manera autónoma y excluyente. No tendría sentido delimitar nuestra actuación a un solo ámbito y mucho menos priorizar uno sobre otro, sino al contrario, y de ahí el objetivo de este artículo: fomentar la interdisciplinariedad, establecer puentes de interacción y dejar las puertas abiertas para que podamos apoyarnos en todas las ciencias posibles que estén relacionadas con nuestros diferentes objetos de estudio. Antropología e Historia. 
La relación entre Antropología e Historia ha caminado de muy diferente manera a lo largo de este último siglo. Si en algunas fases no podríamos distinguir entre ambas, apareciendo la Antropología como una parte de la Historia, en otras etapas del siglo pasado parecían enemistadas y con diferencias insalvables, constituyendo dos ciencias con objetos de estudio marcadamente diferentes y excluyentes para, de nuevo, ya en otras épocas más recientes, volver a estrechar relaciones, articulando puentes de conexión y trabajo común con las que coordinarse y complementarse una a otra. Algunos de estos puentes serían la Historia Oral, la Etnohistoria o la Antropología Histórica.

En un principio, y para tener un punto de partida general con el que exponer nuestros argumentos debemos hacer una breve definición de ambas. Así, entendemos la Antropología como la ciencia que estudia la cultura, entendida ésta como configuradora de las sociedades y elemento fundamental en la composición del ser humano. Para este objeto de estudio, la Antropología emplea como técnica de trabajo la etnografía, basada principalmente en la observación y participación directa con los grupos de estudio en los que corroborar sus hipótesis.

Por otro lado, la Historia se fundamenta como ciencia en el estudio del pasado, "la Historia sigue siendo esencialmente una manera de considerar los datos, y de preguntar y responder a la pregunta ¿por qué? con relación a acontecimientos concretos" (Lewis, 1972, p. 11), empleando soportes materiales como objetos de estudio con los que corroborar sus teorías y construir sus conclusiones.

En esta línea y para comenzar a analizar sus diferencias, la Historia "parece menos interesada en establecer y comprobar generalizaciones acerca de las instituciones sociales que en trazar series de acontecimientos pasados en términos de relación de causa y efecto" (Lewis, 1972, p. 12). Si volvemos la vista a la relación que han mantenido ambas ciencias durante los últimos siglos vemos como aparecen dos conceptos que serán claves para fijar sus diferencias: el objeto de estudio y el tiempo. Que marcarán así los diferentes vaivenes entre ambas.

Partiendo del objeto de estudio y realizando un breve recorrido por la relación entre ambas ciencias, vemos cómo la Antropología acompaña a la Historia desde la Ilustración como ciencia que describe a los "otros", 
distinguiéndolos del "nosotros", que estaría compuesto por el mundo occidental civilizado al que los historiadores ilustrados tienen como objeto de estudio. Es por esto por lo que a la Antropología tradicionalmente se le otorgan los "salvajes" como campo de trabajo. Desde este enfoque, esta ciencia tan sólo sería una epifanía del colonialismo, como ciencia que ocupa este hueco vacante en las otras ciencias, más interesadas en los fenómenos "occidentales".

Desde esta línea, el difusionismo y el evolucionismo imperante a principios de Siglo XX junto al posterior ecologismo, harán que en Estados Unidos, la Antropología se mantenga aunada a la Historia a través de la Prehistoria y la Arqueología, trabajando en los estudios de las tribus noramenrindias; mientras que en Reino Unido se producirá la escisión que caracterizará el devenir de la Antropología a lo largo de todo el Siglo XX y la separación entre ambas ciencias. De la mano del incipiente funcionalismo estructural de A. R. Radcliffe Brown y B. Malinowski - a pesar de W. H. Rivers, como sostiene P. P. Viazzo (Villar, 2004) - los antropólogos se "apropiarán" de esos "otros" como objeto de estudio en exclusiva, distinguiéndose, creando su propio método de trabajo y excluyendo a la Historia por esa falta de documentación de estos grupos, así como por el empleo de otras técnicas de investigación. Separando así, a ambas ciencias de manera excluyente, hasta los años cincuenta que volverán a encontrarse de la mano de E. Evans-Pritchard, quien incidiría en la importancia de la Historia para el conocimiento de la sociedad.

Es por tanto el objeto de estudio la clave para entender la relación entre ambas. El historiador usa objetos materiales que por alguna razón se conservan como testimonio para la posterioridad, mientras que el antropólogo usa la observación y participación directa y personal. De esta forma el antropólogo está sujeto al presente y a la acción constructiva del grupo estudiado, teniendo al pasado y a lo que la Historia dice como base de su documentación; pero en un plano "secundario" ya que le confiere casi el estatus de mito, centrándose en la producción cultural presente para establecer pautas que se cumplan, o al menos que se entiendan y permitan otros estudios en el futuro. Esto provocaría el dilema de la posibilidad del estudio antropológico de grupos desaparecidos o la reconstrucción cultural a 
través de otras fuentes como por ejemplo la Etnoliteratura, debate que sigue abierto en la actualidad.

Tal como dice B. S. Cohn (1962), el historiador tiene que encontrar las fuentes en las que se basa su trabajo, y si no las encuentra tendrá que abandonar la investigación. De hecho, la mayoría de las investigaciones históricas se inician cuando se conocen un conjunto de fuentes históricas. El antropólogo, en cambio, parte de un punto de arranque totalmente distinto. $\mathrm{Su}$ investigación nace de un problema, de un supuesto de hipótesis y a partir de ahí crea los materiales con los que trabajar a medida que va trabajando. Podríamos decir que el antropólogo construye el modelo de sociedad sobre la que va a trabajar, mientras que el historiador inicia su trabajo sobre el modelo de sociedad ya fijado y conocido.

Relacionado con el concepto de objeto de estudio encontramos también la variable del tiempo como elemento separador (o aglutinador) entre ambas ciencias. Si para la Historia la reconstrucción diacrónica resulta fundamental, para la Antropología, el sincronismo de las manifestaciones culturales será, en numerosas investigaciones, su único objetivo del estudio. Así, la perspectiva temporal, la importancia de la reconstrucción cronológica de los hechos, la sincronía o la diacronía en el estudio serán fundamentales para una y otra y marcará el perfil del estudio a desarrollar.

Si para la Historia, la perspectiva diacrónica es primordial, y el continuum entre diferentes momentos pasados se antoja como único elemento con el que entender la causalidad de los hechos y la explicación lógica de su desenlace, para la Antropología resultará secundaria en muchos casos, tratándolos como decíamos más arriba en terminología de mito y centrándose en las instituciones presentes.

Esta dualidad entre pasado y presente, no puede ser de ninguna manera un puente insalvable para ambas ciencias, ya que como se ha visto, a lo largo del siglo XX, por parte de la Antropología, la perspectiva histórica no solo ayuda a entender el devenir de los objetos que se estudian, sino también pueden servir para explicar sus significados, siempre alejados del funcionalismo simplista. Mientras que por el otro lado, la Historia ha entendido que no solo la perspectiva secuencial de los hechos pasados puede servir para explicar los acontecimientos y que se puede (y se debe) apoyar en el enfoque diacrónico con el que la Antropología rellena de cultura esos 
acontecimientos y los dota de una perspectiva holística, completando de esta forma la visión de esos hechos. Si cruzamos ambas visiones, vemos como la Antropología se enriquece con esa perspectiva histórica mientras que la Historia debe añadir la perspectiva cultural, aglutinando en su explicación un componente de emocionalidad (Lorandi, 2012).

Y esto hace que la discusión sobre el rol de ambas ciencias se encamine hacia el carácter de auxiliar que la Antropología ha adoptado en numerosos estudios para la Historia o a la inversa. Siendo éste, creemos, un debate fútil en cuanto que dependerá en exclusiva del atractor que rija la investigación y los protagonistas de ésta, tanto para la obtención de información como por su accesibilidad, viabilidad o interés primario, que hará que se antepongan unos objetivos sobre otros.

Siguiendo las ideas de J. Vansina (1985), considerado como un historiador por los antropólogos y un antropólogo por los historiadores, en sus estudios sobre tradición oral, ya se advierte sobre "la dificultad de comprender cualquier historia si no se ha adquirido antes una familiaridad con la lengua y la cultura de la población de la cual ésta (se supone) representa el pasado" (Villar, 2004, p. 329).

Así y resumiendo la situación hasta la actualidad vemos como tras el devenir de este último siglo, la Antropología ha ayudado a la Historia con el concepto de cultura, ha introducido la emocionalidad en ésta y ha aportado la visión científica de su método de observación para que los historiadores puedan construir una historia fiable a partir de los discursos y las manifestaciones culturales de sus actores protagonistas. Mientras que la Historia ha permitido una mayor comprensión de la encrucijada actual de las instituciones que son objeto de estudio de la Antropología y ha servido de fuente documental con la que situar el inicio de la investigación etnográfica. Recientemente, cuando la Antropología parece haber llegado ya a todos los rincones del planeta y siendo los "antiguos salvajes" los que se han convertidos en antropólogos para describir su propia cultura, aportando otra perspectiva que se antoja definitiva, la Antropología occidental se ha girado hacia sí misma para cambiar el foco de atención y comenzar a trabajar sobre sus propias culturas y sociedades. Los estudios urbanos, movimientos migratorios, relaciones de poder, democracias o análisis de sistemas expertos como la sanidad o educación han pasado a ser los nuevos objetos de estudio 
de la Antropología actual. De esta forma, esta ciencia ha establecido una nueva forma de relacionarse con la Historia, como proveedora de los datos necesarios con los que reconstruir históricamente parte de los fenómenos actuales, dándoles forma cultural y permitiendo su análisis desde otras disciplinas.

Actualmente, hay historiadores que estudian esos "otros" pueblos sin historia para los que las etnografías realizadas por los antropólogos son una herramienta fundamental de estudio, mientras que antropólogos estudiosos de comunidades actuales, necesitan de la perspectiva histórica para entender el funcionamiento actual de ellas.

De esta manera, vemos cómo es interesante su coordinación, tal como dice E. H. Carr "cuanto más sociológica se haga la Historia, y más histórica pase a ser la Sociología, tanto mejor para ambas" (Carr, 1967, p. 66). En esta línea, otros autores que han analizado la vertiente sociológica de la Historia como F. Braudel (1968), P. Burke (1987), J. Casanova (1991), S. Juliá (1989) o J. Reglá (1970) coincidirán en esta perspectiva enriquecedora e interdisciplinar.

Para terminar de clarificar los diferentes conceptos que surgen de esta relación, las ramas o técnicas de trabajo que se establecen entre ambas ciencias han aparecido de la siguiente forma:

La Antropología Histórica, surgirá como una disciplina encargada de reconstruir la historia de los sujetos pasivos insertos en los acontecimientos históricos que la Historia reconstruye, dividiendo los campos de estudio en sociedades con Estado y sin Estado, con escritura o con historia oral.

Es decir, la Antropología Histórica se extenderá por aquellos países que tradicionalmente han sido objeto de estudio para la Historia como meros elementos pasivos, por lo que necesitarán de la perspectiva antropológica para reconstruir su pasado que quedó marginado por esta ciencia. De ahí que surja esta rama de la Antropología, que tendrá mayor protagonismo en los países históricamente colonizados y con fuerte presencia de grupos no incluidos en esa "historia colonial".

Respecto al concepto de Etnohistoria, se entenderá por tanto de manera similar a la Antropología Histórica, desarrollando el patrimonio histórico de los grupos étnicos que por una u otra razón se encuentran insertos en grupos mayoritarios y por lo que no han sido protagonistas de su historia. Quizá esté 
justificado incluirla dentro de la Antropología Histórica, ya que el prefijo etno- invita a delimitar el objeto de estudio a un grupo localizado, haciendo hincapié en ese carácter localista o minoritario, por lo que, se entiende que muchos autores defiendan esa postura de Antropología Histórica como denominación general ${ }^{1}$ (Lorandi, 2012).

Por último, aparece otro concepto relacionado: la historia oral. En los pueblos en los que no existe un soporte material con el que empezar a investigar, el antropólogo puede usar el pasado como mito con el que construir un sistema de creencias. La tradición oral nos puede servir para la investigación histórica como punto de inicio de la investigación o como formulación de la hipótesis con la que partir las investigaciones. Por lo que las herramientas que nos brinda la Antropología a través de la reconstrucción cultural de un pueblo partiendo del conjunto de tradiciones (de su patrimonio), pueden constituir el punto de inicio de una investigación histórica, de ahí la importancia de la historia oral como herramienta de trabajo, especialmente para los etnógrafos.

Tal como nos dice I. M. Lewis (1972, p. 13), la Antropología es una ciencia en la que sus descubrimientos quedan abiertos a posteriores escrutinios y comparaciones, aprovechándose para afirmar o negar teorías de otros colegas. Está dedicada a la elaboración de teorías con las que poder trabajar el resto de investigadores. Es por esto por lo que en la actualidad, la etnografía se puede catalogar como el primer documento de estudio para los historiadores en multitud de ocasiones. Al igual que el periodismo de guerra supone la primera fuente en las contiendas bélicas, la Antropología constituye la primera aproximación a todas las instituciones presentes o carentes de documentación. Tal como rezan los estudios etnográficos, su especialidad es "entrar hasta la cocina" para devolver un texto con rigor científico con el que poder contrastar otras hipótesis ${ }^{2}$. De esta manera, la Historia tiene en la Antropología una ciencia auxiliar con la que trabajar complementariamente, mientras que desde el otro lado, la Antropología cuenta con la visión del historiador para desterrar falsas presunciones o abreviar el trabajo de acercamiento hasta las razones sobre el funcionamiento de las instituciones. 


\section{Las Instituciones como Eje Vertebrador}

Aunque ya hemos adelantado algo sobre la conveniencia del prisma de las instituciones en la conjunción de ambas ciencias, desarrollamos aquí de manera específica esta propuesta a modo de hilo conductor entre ambas ciencias y nuevo aporte al debate entre ambas. Entendemos las instituciones como una de las principales herramientas para el análisis de la configuración cultural de cada una de las sociedades. A modo de redes celulares que componen todo el tejido cultural, cada una de estas células —entendidas a modo de celdillas, tal como afirmaba en sus primeros estudios la Biologíalas instituciones compondrían cada una de esas células en torno a las que los individuos agrupan sus normas y costumbres con las que pautar y facilitar su comportamiento sociocultural. El matrimonio, la familia, la escuela, la religión, el estado o el sistema económico serían algunas de las más evidentes y estudiadas desde esta perspectiva, pero existen muchas más.

Es decir, debido a la respuesta que el ser humano debe dar a sus necesidades biosociales básicas, comenzará a desarrollar unos entes socioculturales que se encargarán de ello - estructurando el comportamiento y dándole forma - y éstos se desarrollarán hasta tal punto que comenzarán a establecerse por sí mismos, formando estructuras propias que se despegarán de ese aparente halo de necesidad básica, pasando a una esfera diferente (cultural). Estos entes serían las instituciones socioculturales, que pasarán de ser organizaciones para resolver las necesidades básicas a pautar ahora cómo van a ser las actividades (y las necesidades) de las sociedades.

De esta manera, las instituciones socioculturales, a pesar de ser esferas colectivas, compartidas y construidas por el grupo, afectan de manera individual a cada individuo, pautando su comportamiento y devenir sociocultural y estructurando los diferentes aspectos del comportamiento grupal e individual.

La importancia de las instituciones radica también en que serán una de las claves para la distinción entre la Historia y la Antropología, atravesadas por esas variables de objeto y tiempo que mencionábamos anteriormente y que dependiendo del enfoque podrán ser — una vez más - elementos aglutinantes o separadores. 
Así, vemos como para la primera, las instituciones discurrirán en etapas sucesivas, irreversibles, no teleológicas y confluyentes en una sucesión que conforma el corpus central de la Historia, con la que reconstruir y narrar tiempos pasados. Mientras que para la Antropología, en cambio, las instituciones estarán definidas como organizaciones de carácter distributivo y serán elementos cíclicos de la sociedad que se relacionan entre sí pero no sucesivamente, poniendo también el foco de interés en las relaciones que se producen entre ellas y marcando la separación entre ambas ciencias.

Hasta ahora, la Antropología era, casi con exclusividad, la encargada de estudiar y diseccionar todos los elementos que componen las instituciones sobre las que estemos estudiando pero no tiene por qué ser así, de hecho, la Historia también las elige como objetos de estudios con los que ordenar su hilo narrativo. Entendiéndolas como estructuras sociales que comprimen las ideas de todos para darle una forma común, con la que establecer el comportamiento socialmente aceptado, con la finalidad de pautar, enseñar, mantener y reproducir los valores culturales del grupo éstas podrán también erigirse como nexo conector entre las ciencias ocupadas de su estudio, situándose a modo de plataforma de actuación conjunta.

De esta forma, las instituciones serán definidas como estructuras que rigen a los grupos sociales, los mercados económicos, los diferentes tipos de poder o los modelos de ciudades imperantes a lo largo de la historia, entre otros muchos ejemplos de su aplicación ${ }^{3}$ y serán el objeto de estudio donde confluyan estas ciencias.

Desde la Antropología, la variedad y forma de clasificar todos los tipos de instituciones que aparecen en las diferentes sociedades (culturas) se hace compleja, relativa y dependiendo del método o enfoque que empleemos mostrará resultados muy diferentes. Tan sólo y a modo de exposición aclaratoria, podríamos mostrar algunos tipos de instituciones con las que ilustrar nuestro posterior análisis. La familia, el estado, la escuela, el idioma, el derecho o incluso la moda serían algunas de las instituciones objeto de análisis por parte de la Antropología. Ahora bien, una posible clasificación de éstas, las podría agrupar en primarias y secundarias, tal como hace A. Kardiner (1974 [1939]), o según los campos principales de su estudio como instituciones sociales, políticas, religiosas, económicas, etc. También podemos encontrar otra clasificación diferente según su función, como 
instituciones de transición, de entrada o de salida. Atendiendo al tipo de poder imperante, desde el prisma de la Antropología Política y Económica podríamos encontrar instituciones centralizadas y no-centralizadas; respecto a su ámbito podemos encontrarlas públicas, privadas o mixtas, según su espacio, locales, nacionales, internacionales e incluso atendiendo a su nivel de complejidad, instituciones sencillas o complejas (expertas).

Del mismo modo, encontramos las funciones que éstas desempeñan dentro de la sociedad como herramientas para fijar y controlar el comportamiento del grupo, podemos pensar en la familia, la religión o algunas de transición como los estudios superiores, que servirán para someter al individuo a un conjunto de reglas con las que pautar y modelar su comportamiento dentro del grupo o para hacerlo progresar hacia un comportamiento determinado y aceptado por el resto. Tanto la aceptación como el rechazo de estas instituciones formarán parte del modelo cultural que plantea el grupo, que acepta — en el mismo sentido que tomamos la cultura - las instituciones, las vive y las transforma. Es decir, al igual que la cultura se convierten en acción y proceso.

En este sentido, podemos intuir ya la convergencia de la Historia y la Antropología bajo este prisma. Si añadimos a la primera como ciencia que también convergerá en el análisis de las instituciones socioculturales, observando cómo las funciones de las instituciones socioculturales vendrán determinadas por el carácter que el propio grupo les infunda, las tendremos que analizar desde dentro, de la misma manera que cualquier otra esfera de la cultura, con una perspectiva reflexiva y holística y por supuesto histórica. Las instituciones sirven para socializar y controlar al sujeto, nos invisten de un habitus para que consigamos nuestro rol social y lo desempeñemos correctamente, al tiempo que secuenciará nuestro ritmo biográfico (Jacinto \& Millenaar, 2009) a lo que podríamos añadir que constituirán el eje vertebrador del correlato científico que realizarán diferentes ciencias, entre ellas, la Historia y la Antropología.

No resulta difícil desde esta perspectiva ver cómo las instituciones serán el verdadero nexo conector para las investigaciones actuales en las que la Historia y la Antropología converjan. Utilizando este prisma podemos intuir la importancia de la perspectiva antropológica para su comprensión y 
obtención de datos y exactamente igual a la inversa, lo fundamental de la Historia en la comprensión de la situación actual del objeto de estudio.

Es por esto por lo que planteamos aquí el enfoque de las instituciones socioculturales como nexo de unión entre ambas ciencias en las que confluirán tanto el tiempo como el objeto. Ya sea desde una perspectiva sucesiva y teleológica para la Historia como concéntrica y diacrónica para la Antropología, en el caso del análisis de las instituciones, desde el prisma del tiempo. O desde la disección de su objeto de acción e influencia, como institución vertebradora y causal, desde un punto de vista funcionalista en su análisis histórico o como constitución cultural dentro de la sociedad, desde la perspectiva antropológica.

Bajo esta perspectiva, las instituciones socioculturales contemporáneas se erigen como un buen ejemplo de objeto común en la convergencia de ambas ciencias.

\section{Instituciones Socioculturales Contemporáneas}

Si tomamos las instituciones socioculturales contemporáneas como marco en el que aplicar todo lo expuesto anteriormente vemos como existen algunos perfiles que, debido a su particularidad, ya sea por la dificultad en el acceso, su reciente creación o la falta de documentación producida, se convierte en elementos idóneos en los que aplicar este cruce de caminos con los que demostrar la utilidad de la Antropología y la Historia como ciencias coordinadas y bidireccionales.

En este sentido, siguiendo la clasificación sobre las diferentes instituciones socioculturales realizada por Berdichewsky (2002, p. 87) y mencionada brevemente más arriba, podemos mostrar algunos ejemplos en el estudio de las instituciones relacionadas con el mantenimiento físico instituciones económicas, modos de producción, (dinero, sectores productivos, organización socioeconómica, etc.) - donde hay algunos estudios en este sentido desde la Antropología como los realizados por P. Moreno Feliú (2012) y su análisis económico de los campos de concentración o los de I. Moreno Navarro (1999) sobre las culturas del trabajo e identidad en los que la perspectiva histórica resultará fundamental. De la misma forma otros como el estudio de la bolsa de valores de E. Hertz 
(1999) o K. Ho (2009) en los que Historia y Antropología se entrecruzan en el análisis de las instituciones económicas actuales nos muestran esta perspectiva.

En las instituciones que regulan la vida sexual, procreación y mantenimiento (familia, matrimonio, parentesco, construcción de géneros, etc.) aparecen estudios como los ya clásicos de M. Mead (1985 [1929]) que tendrán vigencia en los análisis de las instituciones actuales; los estudios etnográficos sobre la familia de M. Segalem (1987) o a la inversa, el devenir histórico de la familia según A. Burguiere (1986) que será tan útil a la Antropología.

Encontramos también otros ejemplos de esta bidireccionalidad en las instituciones relacionadas con la sociabilidad, socialización y endoculturación (escuela, grupo sociales, entidades educativas, etc.) donde podemos mencionar estudios como el de J. L. Anta Félez (1990) sobre cuarteles militares o C. Feixá sobre tribus urbanas, y a la inversa, la perspectiva histórica tan útil para la Antropología en el análisis histórico de las instituciones educativas por parte de O. Negrín Fajardo (2009) y el devenir de la educación.

$\mathrm{Si}$ tomamos como ejemplo estas instituciones, entendidas como estructuras de transición, vemos como también sirven para facilitar el abandono de una situación social y el ingreso en otro nuevo. De este modo, el paso a la vida adulta vendría secuenciado por instituciones como el sistema educativo, la familia o el trabajo, con los que asegurar la entrada y aceptación del nuevo estatus. En esta línea, estudios desde la Antropología como los de la autora C. Jacinto (2009) o M. I. Jociles y A. Franzé (2008), sobre el descrédito que sufren actualmente algunas de estas instituciones como la escuela, la familia o el trabajo entrecruzarían la perspectiva histórica, antropológica y, en estos casos, también la sociológica.

Este fenómeno de la decadencia o crisis de las instituciones es un tema ampliamente analizado en la actualidad. Al igual que en otros campos, los constantes cambios que se producen a nuestro alrededor, provocan la reflexión y el análisis; y las instituciones se han convertido en una buena herramienta para su comprensión y estudio, al tiempo que se produce un meta-análisis sobre cómo funcionan y piensan las propias instituciones, dotándolas de esta manera, de una identidad propia y ajena al grupo, 
actuando en algunas ocasiones aparentemente incluso en su contra. De esta manera, las instituciones socioculturales se convierten en una buena herramienta con la que fusionar, ya no solo la Historia y la Antropología, sino otras disciplinas como la Sociología o la Psicología.

Las instituciones creadas en torno al control social (instituciones jurídicopolíticas, liderazgo, tomas de decisión, modos de legitimación, procesos políticos) son otro ejemplo de esta conjunción de las ciencias donde aparecen los múltiples estudios que van desde el análisis del congreso estadounidense a semejanza de una tribu (Mclver Weatherford, 1985) hasta los estudios sobre identidad nacional y las diferentes percepciones de concepto de estado, nación o pueblo estudiadas por T. Lewellen (1994) en la que ambas ciencias se vuelven a encontrar.

Aquí también aparecen otras instituciones culturales en las que ponen el foco ambas ciencias y se compaginan bajo esta perspectiva, así, la guerra como institución humana y su análisis contemporáneo, tal como hace $\mathrm{K}$. Otterbein (2009) sumado a todos los efectos que produce: movimientos migratorios, modificación de fronteras, etc. serán otras de las instituciones en las que confluyan ambas ciencias; otras de carácter secundario que también nos servirán como ejemplo de coordinación a través de esta perspectiva serán los estudios sobre la moda, trajes y representación del poder (Barañano \& Cátedra, 2005), la imagen (Belting, 2007) o los estudios sobre las nuevas redes sociales e internet (Estalella \& Ardévol, 2010).

Todo esto nos demuestra cómo se presentan una amalgama de variables que nos permiten refutar lo anterior al tiempo que nos muestran por donde caminan las tendencias investigativas actuales. Así vemos como la Antropología y la Historia deben coordinarse, vemos como la etnografía arroja datos suficientes para que el historiador los interprete y a la inversa, el antropólogo se nutre de todos los estudios realizados desde la Historia para situarse en un punto de partida suficientemente ventajoso como para que su trabajo etnográfico sea provechoso.

De la misma manera, en el resultado final, deberá encajar tanto el enfoque histórico como antropológico con el que situar un fotograma más en la reconstrucción científica de las instituciones socioculturales, que se erigen así como otro elemento vertebrador bajo el que tender puentes de unión entre ambas ciencias. 
En esta línea, las aportaciones de la Historia a la Antropología, o por qué usar la perspectiva histórica en un estudio etnográfico de Antropología sobre instituciones socioculturales, resulta de sencilla respuesta, ya que sería inexcusable obviar la perspectiva histórica para entender su devenir y entender que los procesos de cambio que estos sufren están siendo paulatinos y paralelos a la demanda social en la que están insertos, por lo que también hay que entenderlos en su propia encrucijada histórica, la cual hay que conocer ya que dará respuesta a multitud de preguntas previas al trabajo etnográfico. Y justo a la inversa, la Historia necesita de la Antropología para poder iniciar una investigación sobre las instituciones contemporáneas, de carácter cambiante y de las que haya poco escrito debido a su reciente creación. Por lo que la etnografía supone un documento eficaz con el que comenzar a entender su situación actual y el rumbo que posiblemente irán tomando. En definitiva, serán el conocimiento de las instituciones y la denominación de éstas como herramientas con las que diseccionar y analizar nuestro objeto de estudio las que nos permitan tender puentes de entendimiento entre ambas ciencias.

\section{Notas}

${ }^{1}$ Cfr. ¿Etnohistoria, Antropología Histórica o simplemente Historia? (Lorandi, 2012).

${ }^{2}$ Cfr. Etnografía. Métodos de investigación (Hammersley \& Atkinson, 2005) o Cultura, Antropología y otras tonterías (Díaz de Rada, 2012).

${ }^{3}$ No hemos creído necesario incluir una revisión histórica sobre el estudio de las teorías de las instituciones, que podríamos comenzar con E. Burke, Bonald y De Maistre en el siglo XIX hasta los recientes estudios de M. Douglas (1996a), N. Luhman (1990) pasando por autores del siglo XX como Gehlen (1987 [1940]), L. A. Coser (1978), M. Foucault (1978), E. Goffman (2001 [1961]) u otros autores dentro de la Filosofía y la Sociología que también aportarán su revisión y tratarán este tema, como K. Marx, J. Habermas, K. Popper o H. Marcuse (Cfr. Teorías conservadoras y teorías críticas de las ciencias sociales) (Vergara, 2001). 
142 Alcalde Sánchez - Antropología, Historia, Instituciones Socioculturales

\section{Bibliografía}

Anta Félez, J. L. (1990). Cantina, garita, cocina. Estudio antropológico de soldados y cuarteles. Madrid: Siglo XXI Editores.

Barañano, A., \& Cátedra, M. (2005). La representación del poder y el poder de la representación. Política y sociedad, 42(3), 227-250.

Belting, H. (2007). Antropología de la imagen. Buenos Aires: Katz Editores. Berdichewsky, B. (2002). Antropolgía Social: Introducción. Una visión global de la humanidad. Santiago: LOM Ediciones.

Braudel, F. (1968). La Historia y las Ciencias Sociales. Madrid: Alianza. Bueno, G. (26 de enero de 2010). Instituciones. Recuperado del perfil de fgbuenotv: https://www.youtube.com/watch?v=GDr10mXJpwo Burguiere, A., Klaspich, K., \& Zonabend, F. (1986). Historia de la familia.

Madrid: Alianza.

Burke, P. (1987). Sociología e Historia. Madrid: Alianza.

Cámara Arroyo, S. (2010). La finalidad educativa de los Centros de Internamiento de Menores: el Hospicio como antecedente. Anuario Facultad de Derecho - Universidad de Alcalá III, 521 - 554.

Carr, E.H. (1967). ¿Qué es la Historia? Barcelona: Seix Barral.

Casanova, J. (1991). La historia social y los historiadores. Barcelona:

Editorial Crítica.

Cohn, B.S. (1962). Un antropólogo entre historiadores. Un informe de campo. Oxford: Oxford University Press.

Coser, L.A. (1978). Las instituciones voraces: visión general. Fondo de Cultura Económica: Buenos Aires.

Cruces Villalobos, F., \& Pérez Galán, B. (2011). Textos de Antropología Contemporánea. Madrid: UNED.

Díaz de Rada, Á. (2012). Cultura, Antropología y otras tonterías. Madrid: Trotta.

Douglas, M. (1996a). Cómo piensan las instituciones. Madrid: Alianza Editorial.

Douglas, M. (1996b). La aceptabilidad del riesgo según las ciencias sociales. Barcelona: Paidós. 
Estalella, A., \& Ardévol, E. (2010). Internet: instrumento de investigación y campo de estudio para la antropología visual. Revista chilena de antropología visual, 15, 1-21.

Fairchild, H.P. (1949). Diccionario de Sociología. México: Fondo de Cultura Económica FCE.

Foucault, M. (1978). La voluntad del saber. México D.F.: Siglo XXI Editores.

Geertz, C. (2003[1973]). La interpretación de las culturas. Barcelona: GEDISA.

Gehlen, A. (1987 [1940]). El hombre, su naturaleza y su lugar en el mundo. Salamanca: Sígueme.

Goffman, E. (2001 [1961]). Internados. Ensayo sobre la situación social de los enfermos mentales. Buenos Aires: Amorrortu Editores.

Hammersley, M., \& Atkinson, P. (2005). Etnografía. Metodos de investigación. Barcelona: Paidós.

Hertz, E. (1999). The Trading Crowd: An Ethnography of the Shanghai Stock Market. Cambridge: Cambridge University Press.

Ho, K. (2009). Disciplining Investment Bankers, Disciplining the Economy:

Wall Street's Institutional Culture of Crisis and the Downsizing of 'Corporate America. American Anthropologist 111(2) 177-189. doi:

https://doi.org/10.1111/j.1548-1433.2009.01111.x

Jacinto, C., \& Millenaar, V. (2009). Enfoques de programas para la inclusión laboral de los jóvenes pobres. Lo institucional como soporte subjetivo. Revista Última Década , 67-92.

Jociles, M.I., \& Franzé, A. (2008). ¿Es la escuela el problema?Perspectivas socio antropológicas de etnografía y educación. Madrid: Editorial Trotta.

Juliá, S. (1989). Historia social/sociología histórica. Madrid: Siglo XXI. Kardiner, A., \& Linton, R. (1974 [1939]). The Individual and his Society: the Psychodynamics of Primitive Social Organization. Virginia: Greenwood Publishing Group.

Lewellen, T. C. (1994). Introducción a la antropología política. Barcelona: Edicions Bellaterra.

Lewis, I.M. (1972). Historia y Antropología. Barcelona: Seix Barral. 
Lorandi, A.M. (2012). ¿Etnohistoria, Antropología Histórica o simplemente Historia? Memoria Americana. Cuadernos de Etnohistoria, 17-34. Luhmann, N. (1990). Sociedad y sistema: la ambición de la teoría. Barcelona: Paidós.

Mclver Weatherford, J. (1985). Tribes on the Hill. The U.S. Congress, rituals and realities. New York: Bergin \& Garvey Publishers. Mead, M. (1985 [1929]). Adolescencia, sexo y cultura en Samoa. Barcelona: Planeta.

Montero Pedrera, A.M. (2008). Luis Amigó y Ferrer, Los terciarios capuchinos y la protección de menores. Escuela Abierta, 167-189. Moreno Feliú, P. (2012). En el corazón de la zona gris. Una lectura etnográfica de los campos de Auschwitz. Madrid: Editorial Trotta. Moreno Navarro, I. (1999). Globalización, ideologías sobre el trabajo y culturas del trabajo. Areas: Revista internacional de ciencias sociales, 19, 17-34.

Negrín Fajardo, O., \& Vergara Ciordia, J. (2009). Historia de la Educación. Madrid: Editorial universitaria Ramón Areces.

Otterbein, K. (2009). Anthropology of the war. Long Grove: Waveland Press.

Petit, F. (1984). Psicosociología de las organizaciones: introducción a sus fundamentos teóricos y metodológicos. Barcelona: Herder. Reglá, J. (1970). Introducción a la Historia: socioeconomía, política, cultura. Barcelona: Teide.

Sánchez Vázquez, V., \& Guijarro Granados, T. (2002). Apuntes para una historia de las instituciones de menores en España. Revista Asociacion Española de Neuropsiquiatría , XXII(84), 121 - 138.

Schmitt, C. (1991). El concepto de lo político. Madrid: Alianza.

Segalem, M. (1987). Antropología histórica de la familia. Madrid: Taurus. Valdés del Toro, R., \& Valdés Gasquez, M. (1989). Instituciones sociales.

En E. U. Social, Treball social. Conceptes y eines bàsiques (pp. 103112). Barcelona: Institut Catòlic d' Estudis Socials de Barcelona.

Vansina, J. (1985). Oral tradition as history. Oxford: James Currey Publishers.

Vergara, J.I. (2001). Teorías conservadoras y teorías críticas de las instituciones sociales. Revista Ciencias Sociales, 138-157. 
Viazzo, P.P. (2003). Introducción a la Antropología Histórica. Lima: Pontificia Universidad Católica de Perú.

Villar, D. (2004). Viazzo, Pier Paolo. Introducción a la Antropología Histórica. Lima: Pontificia Universidad Católica del Perú, Instituto Italiando de la Cultura. Revista de Antropología , 47 (1), 325 - 329. Virno, P. (2011). Ambivalencia de la multitud. Entre la innovación y la negatividad. Buenos Aires: Tinta Limón Ediciones.

Ignacio Alcalde Sánchez es profesor de Antropología Social en el departamento de Ciencias Sociales y Humanidades de la Universidad de Córdoba y profesor del CIMI Sierra Morena.

Dirección de contacto: Facultad de Filosofía y Letras, Universidad de Córdoba, Plaza del Cardenal Salazar, 3, 14071 Córdoba, España.

Email: ialcalde@uco.es 